\section{Horizontal Versus Longitudinal Axis of the Hippocampus: Metabolic Differentiation as Measured with High-Resolution PET/MRI}

TO THE EDITOR: With great interest we read the recent article by Cho et al. entitled "Substructural Hippocampal Glucose Metabolism Observed on PET/MRI" (1). In that work the authors performed high-resolution ${ }^{18} \mathrm{~F}$-FDG PET and structural MRI of the hippocampus in a group of 5 healthy subjects. Among the hippocampal subfields, the highest ${ }^{18} \mathrm{~F}$-FDG uptake was consistently found in the dentate gyrus and cornu ammonis (CA) 4. This report is the first such structural/functional study benefiting from the latest advances in spatial resolution and is therefore of special relevance. However, we wish to suggest an additional data analysis, which from our point of view is essential to implicitly address the issue raised by the authors, that is, substructural hippocampal glucose metabolism.

The hippocampus is a limbic region with a very complex anatomy and function. Based on the predominant cell type, cell origin, and connectivity, the hippocampus is typically segmented into the dentate gyrus; CA1, CA2, CA3, and CA4 fields; and subiculum. This organization, which roughly fits the horizontal axis of the structure, is considered fundamental to hippocampal function, and numerous studies have focused on the above cytoarchitectural subdivisions to explain functional specialization within the hippocampus. Growing evidence indicates, however, the presence of functional differentiation also along the longitudinal, or anteroposterior, axis of the hippocampus. This concept, initially based on animal data, has recently been supported by in vivo neuroimaging studies. Specifically, several investigators have reported a relationship between neuroimaging indices of the anterior hippocampus and episodic memory performance (2-4).

As for ${ }^{18}$ F-FDG PET, Ouchi et al. (5) found glucose metabolism to be higher in the head than in the body or tail parts of the hippocampus in a group of 8 healthy subjects (mean age of $58 \mathrm{y}$ ). In contrast, patients with pure amnesia or with mild Alzheimer dementia showed significantly decreased metabolism in the hippocampal head only. The authors of that study suggested that the energy consumption subserving synaptic transmission through the perforant pathway was higher in the anterior hippocampus, so as to maintain appropriate cognitive functions such as episodic memory. They also claimed that metabolic depression in this region can cause the memory dysfunction seen in amnestic patients. Indeed, the principal input from multimodal association neocortical areas occurs mainly in the anterior parts of the hippocampus, through the perforant pathway arising in the entorhinal cortex. Integrity of the perforant path is a major prerequisite for normal memory function, such that disruption of this path, as occurs very early in the course of Alzheimer disease, causes profound memory impairment (6).

Unfortunately, Cho et al. did not mention the age of their subjects. This is important, as the hippocampal subfields are differently prone to age-dependent processes (7), although the lack of atrophy in the MR images suggests that the subjects were

COPYRIGHT @ 2011 by the Society of Nuclear Medicine, Inc. relatively young. More importantly, the authors did not assess glucose metabolism along the longitudinal axis of the hippocampus, that is, separately for the hippocampal head, body, and tail. Such data would be of great interest for understanding the neurobiology of hippocampal function, as the rank order of metabolic rates might prove to be more distinct along the longitudinal axis of the hippocampus than along the horizontal axis investigated in the study.

Although hippocampal glucose metabolism has been already mapped by Ouchi et al. (5), the improved spatial resolution of the instrumentation possessed by Cho et al. presents distinct advantages. Specifically, the lesser effects of partial volume in the ${ }^{18}$ F-FDG images, and the nearly perfect coregistration afforded by the hybrid PET/MRI system, make their approach particularly appropriate for studying hippocampal metabolism at the substructural level.

\section{REFERENCES}

1. Cho Z-H, Son Y-D, Kim H-K, et al. Substructural hippocampal glucose metabolism observed on PET/MRI. J Nucl Med. 2010;51:1545-1548.

2. Chetelat G, Desgranges B, de la Sayette V, et al. Dissociating atrophy and hypometabolism impact on episodic memory in mild cognitive impairment. Brain. 2003;126:1955-1967.

3. Chua EF, Schacter DL, Rand-Giovannetti E, Sperling RA. Evidence for a specific role of the anterior hippocampal region in successful associative encoding. Hippocampus. 2007;17:1071-1080.

4. Yakushev I, Müller MJ, Lorscheider M, et al. Increased hippocampal head diffusivity predicts impaired episodic memory performance in early Alzheimer's disease. Neuropsychologia. 2010;48:1447-1453.

5. Ouchi Y, Nobezawa S, Okada H, Yoshikawa E, Futatsubashi M, Kaneko M. Altered glucose metabolism in the hippocampal head in memory impairment. Neurology. 1998;51:136-142.

6. Hyman BT, Van Hoesen GW, Kromer LJ, Damasio AR. Perforant pathway changes and the memory impairment of Alzheimer's disease. Ann Neurol. 1986; 20:472-481.

7. West MJ, Coleman PD, Flood DG, Troncoso JC. Differences in the pattern of hippocampal neuronal loss in normal ageing and Alzheimer's disease. Lancet. 1994;344:769-772.

Igor Yakushev*

Andreas Fellgiebel

*Department of Psychiatry and Psychotherapy

University Medical Center Mainz.

Untere Zahlbacher Strasse 8

55131 Mainz, Germany

E-mail: igoryakushev@uni-mainz.de

DOI: 10.2967/jnumed.110.085084

REPLY: We very much appreciate the comments and suggestions of Yakushev and Fellgiebel on our paper (1), which was the first in a series we are publishing on in vivo human molecular imaging using our newly introduced ultra-high-resolution PET-MRI fusion system (2). Like Yakushev et al. (3), we have also noticed the importance of longitudinal differential metabolic activities, and we are preparing several experiments along that line. The results of some of these have already been reported (4). 